\title{
The countable existentially closed pseudocomplemented semilattice
}

\author{
Joël Adler
}

Received: date / Accepted: date

\begin{abstract}
As the class $\mathcal{P C S} \mathcal{L}$ of pseudocomplemented semilattices is a universal Horn class generated by a single finite structure it has a $\aleph_{0}$-categorical model companion $\mathcal{P C S} \mathcal{L}^{*}$. As $\mathcal{P C S} \mathcal{L}$ is inductive the models of $\mathcal{P C S} \mathcal{L}^{*}$ are exactly the existentially closed models of $\mathcal{P C S} \mathcal{L}$. We will construct the unique existentially closed countable model of $\mathcal{P C S} \mathcal{L}$ as a direct limit of algebraically closed pseudocomplemented semilattices.
\end{abstract}

Keywords Existentially closed · Pseudocomplemented semilattice · Model companion $\cdot \aleph_{0}$-categoricity

Mathematics Subject Classification (2010) 03C05 - 03C10 - 06D15

\section{Introduction}

For a first-order language $\mathcal{L}$ and an $\mathcal{L}$-structure $\mathbf{M}$ with universe $M$ the language $\mathcal{L}(M)$ is obtained by adding a constant symbol for every $m \in M$. To define the notion of model companion we first have to define the notion of model completeness. An $\mathcal{L}$-theory $T$ is said to be model complete if for every model $\mathbf{M}$ of $T$ the set of $\mathcal{L}$-sentences $T \cup \operatorname{diag}(\mathbf{M})$ is complete, where $\operatorname{diag}(\mathbf{M})$ is the set of atomic and negated atomic $\mathcal{L}(M)$-sentences that hold in $\mathbf{M}$. $T^{*}$ is said to be a model companion of $T$ if (i) every model of $T^{*}$ is embeddable in a model of $T$ and vice versa and (ii) $T^{*}$ is model complete.

A theory $T$ need not have a model companion as is the case for the theory of groups and theory of commutative rings, see Wheeler [9]. However, if $T$ is a set of Horn sentences and the class $\operatorname{Mod}(T)$ of its models is finitely generated then $T$ has a model companion $T^{*}$ as was shown by Burris and Werner [4].

J. Adler

Pädagogische Hochschule Bern, Fabrikstrasse 8, 3012 Bern, Switzerland

Tel.: +41-31-3092452

Fax: +41-31-3092411

E-mail: joel.adler@phbern.ch 
If $T$ is additionally inductive - that is $\operatorname{Mod}(T)$ is closed under the union of chains - then we have the characterization $\operatorname{Mod}\left(T^{*}\right)=\operatorname{Mod}(T)^{e c}$, that is, the models of $T^{*}$ are the existentially closed models of $T$, see Macintyre [7. A definition of the notions of algebraically and existentially closed can be found in [1. Finally, if $\operatorname{Mod}(T)$ is generated by a single finite structure then $\operatorname{Mod}\left(T^{*}\right)$ is $\aleph_{0}$-categorical, see Burris [3].

Horn and Balbes [2] proved that $\mathcal{P C S} \mathcal{L}$ is equational, Jones [6] showed that it is generated by a single finite structure. Thus $\mathcal{P C S \mathcal { L } ^ { * }}$ is $\aleph_{0}$-categorical and its only countable model is the countable existentially closed pseudocomplemented semilattice.

In Section 2 we provide the basic properties and algebraic notions concerning pseudocomplemented semilattices (p-semilattices for short), while in Section 3 the countable existentially closed p-semilattice is constructed.

\section{Basic properties of pseudocomplemented semilattices and notation}

A p-semilattice $\left\langle P ; \wedge,{ }^{*}, 0\right\rangle$ is an algebra where $\langle P ; \wedge\rangle$ is a meet-semilattice with least element 0 , and for all $x, y \in P, x \wedge y=0$ if and only if $y \leq x^{*}$ $(x \leq y$ is defined to hold for $x, y \in P$ if $x \wedge y=x$ ).

From the above definition, it follows that in a p-semilattice the following properties hold:

$$
\begin{aligned}
x \leq y & \Longrightarrow y^{*} \leq x^{*} \\
x & \leq x^{* *} \\
x^{*} & =x^{* * *}
\end{aligned}
$$

$1:=0^{*}$ is obviously the greatest element of $P . x \| y$ is defined to hold if neither $x \leq y$ nor $y \leq x$. An element $d$ of $P$ satisfying $d^{*}=0$ is called dense, and if additionally $d \neq 1$ holds, then $d$ is called a proper dense element. For $\mathbf{P} \in \mathcal{P C} \mathcal{S} \mathcal{L}$ the set $\mathrm{D}(\mathbf{P})$ denotes the subset of dense elements of $\mathbf{P},\langle\mathrm{D}(\mathbf{P}) ; \wedge\rangle$ being a filter of $\langle P ; \wedge\rangle$. An element $s$ is called skeletal if $s^{* *}=s$. The subset of skeletal elements of $\mathbf{P}$ is denoted by $\operatorname{Sk}(\mathbf{P})$. The abuse of notation $\operatorname{Sk}(x)$ for $x \in \mathrm{Sk}(\mathbf{P})$ and $\mathrm{D}(x)$ for $x \in \mathrm{D}(\mathbf{P})$ should not cause ambiguities. Equation (3) implies $\operatorname{Sk}(\mathbf{P})=\left\{x^{*}: x \in P\right\}$. In $\operatorname{Sk}(\mathbf{P})$ the supremum of two elements exists with $\sup _{\mathrm{Sk}}\{a, b\}=\left(a^{*} \wedge b^{*}\right)^{*}$ for $a, b \in \operatorname{Sk}(\mathbf{P})$. Instead of $\sup _{\mathrm{Sk}}\{a, b\}$ we use the shorter $a \dot{\vee} b$, assuming $a, b \in \operatorname{Sk}(\mathbf{P})$, which follows from (1) and (2). Observe that $\left\langle\operatorname{Sk}(\mathbf{P}) ; \wedge, \dot{V},{ }^{*}, 0,1\right\rangle$ is a boolean algebra.

For any p-semilattice $\mathbf{P}$ the p-semilattice $\widehat{\mathbf{P}}$ is obtained from $\mathbf{P}$ by adding a new top element. The maximal proper dense element of $\widehat{\mathbf{P}}$ is denoted by $e$. Furthermore, the p-semilattices $\widehat{\mathbf{B}}$ with $\mathbf{B}$ being a boolean algebra interpreted as a p-semilattice are exactly the subdirectly irreducible p-semilattices. Moreover, let $\mathbf{2}$ denote the two-element boolean algebra and $\mathbf{A}$ the countable atomfree boolean algebra. 
An equational set $\Sigma$ of axioms for $\mathcal{P C S} \mathcal{L}$ can be found in [1, for more background on p-semilattices in general consult Frink [5] and [6].

In Schmid [8] the following characterization of algebraically closed p-semilattices is established:

Theorem 1 A p-semilattice $\mathbf{P}$ is algebraically closed if and only if for any finite subalgebra $\mathbf{F} \leq \mathbf{P}$ there exists $r, s \in \mathbb{N}$ and a $p$-semilattice $\mathbf{F}^{\prime}$ isomorphic to $\mathbf{2}^{r} \times(\widehat{\mathbf{A}})^{s}$ such that $\mathbf{F} \leq \mathbf{F}^{\prime} \leq \mathbf{P}$.

In [1] the following (syntactic) description of existentially closed p-semilattices is given:

Theorem 2 A p-semilattice $\mathbf{P}$ is existentially closed if and only if $\mathbf{P}$ is algebraically closed and satisfies the following list of axioms:

(EC1)

(EC2)

$$
\left(\forall b_{1}, b_{2} \in \operatorname{Sk}(\mathbf{P})\right)\left(\exists b_{3} \in \operatorname{Sk}(\mathbf{P})\right)\left(b_{1}<b_{2} \longrightarrow b_{1}<b_{3}<b_{2}\right)
$$

$$
\begin{aligned}
&\left(\forall b_{1}, b_{2} \in \operatorname{Sk}(\mathbf{P}), d \in \mathrm{D}(\mathbf{P})\right)\left(\exists b_{3} \in \operatorname{Sk}(\mathbf{P})\right)( \\
&\left(b_{1} \leq b_{2}<d<1 \quad \& \quad b_{1}^{*} \| d\right) \\
&\left.\longrightarrow\left(b_{2}<b_{3}<1 \quad \& \quad b_{1}^{*} \wedge b_{3} \| d \& b_{1} \dot{\vee} b_{3}^{*}<d\right)\right),
\end{aligned}
$$

$$
\begin{gathered}
(\forall b \in \mathrm{Sk}(\mathbf{P}) \backslash\{1\})(\exists d \in \mathrm{D}(\mathbf{P}))\left(b<d \quad \& \quad b^{*} \| d\right), \\
\left(\forall d_{1}, d_{2} \in \mathrm{D}(\mathbf{P})\right)\left(\exists d_{3} \in P\right)\left(d_{1}<d_{2} \longrightarrow\left(d_{1}<d_{3}<d_{2}\right)\right),
\end{gathered}
$$

$$
\begin{aligned}
\left(\forall b \in \mathrm{Sk}(\mathbf{P}), d_{1} \in \mathrm{D}(\mathbf{P})\right) & \left(\exists d_{2} \in \mathrm{D}(\mathbf{P})\right)\left(0<b<d_{1}\right. \\
& \left.\longrightarrow\left(d_{2}<d_{1} \quad \& \quad b \| d_{2} \& d_{1} \wedge b^{*}=d_{2} \wedge b^{*}\right)\right) .
\end{aligned}
$$

Constructing the unique countable model of the model companion $\Sigma^{*}$ of $\mathcal{P C S} \mathcal{L}$ thus amounts to constructing a countable algebraically closed psemilattice that satisfies (EC1) (EC5).

\section{The construction}

As the objects of the direct limit we are going to construct we take $\left\{\mathbf{G}_{n}: n \in\right.$ $\mathbb{N} \backslash\{0\}\}$, where $\mathbf{G}_{n}:=(\widehat{\mathbf{A}})^{n}$. In view of Theorem $1 \mathbf{G} \mathbf{G}_{n}$ is algebraically closed for all $n \in \mathbb{N} \backslash\{0\}$. We have to define embeddings $f_{n}: \mathbf{G}_{n} \rightarrow \mathbf{G}_{n+1}$ for $n \geq 1$ such that the direct limit of the directed family $\left\{\left\langle\mathbf{G}_{m}, g_{m, n}\right\rangle: m, n \in \mathbb{N}, 1 \leq m \leq n\right\}$ where $g_{i, j}:=f_{j-1} \circ \cdots \circ f_{i}$ for $i<j$ and $g_{i, i}=\operatorname{id}_{G_{i}}$ additionally satisfies (EC1) (EC5) of Theorem 2. The elements of the direct limit are defined by considering an equivalence relation on $\bigcup_{i=1}^{\infty} G_{i} .\left(x_{1}, \ldots, x_{m}\right)$ and $\left(y_{1}, \ldots, y_{n}\right)$ are in the same equivalence class if $m=n$ or $m<n$ and $g_{m, n}\left(x_{1}, \ldots, x_{m}\right)=\left(y_{1}, \ldots, y_{n}\right)$.

We will show that (EC1) (EC5) hold if we have the following properties: 
(P1) For every anti-atom $d$ of $\mathrm{D}\left(\mathbf{G}_{n}\right)$ there is a $k \in \mathbb{N}$ such that $g_{n, n+k}(d)$ is not an anti-atom of $\mathbf{G}_{n+k}$ anymore. This will imply that the order restricted to the dense elements of the direct limit is dense.

(P2) For every $a \in \operatorname{Sk}\left(\mathbf{G}_{n}\right) \backslash\{0,1\}$ there exists $k \in \mathbb{N}$ such that $\pi_{n+k}\left(g_{n, n+k}(a)\right)=$ 1.

To define the embeddings $f_{n}$ we consider the following functions.

(1) Let $r: \mathbb{N} \backslash\{0\} \rightarrow \mathbb{N} \backslash\{0\}$ be such that

- $r(i) \leq 2 i$ for all $i$,

- $r^{-1}(j)$ is infinite for all $j$.

(2) Let $s: \mathbb{N} \backslash\{0\} \rightarrow(\widehat{A} \backslash\{0, e, 1\}) \times(\mathbb{N} \backslash\{0\})$ be such that

- $\pi_{2}((s(i)) \leq 2 i+1$ for all $i$,

- $s^{-1}(a, j)$ is infinite for all $(a, j)$.

(3) Let $U$ be a function with domain $\widehat{A} \backslash\{0, e, 1\}$ which assigns to each $\widehat{A} \backslash$ $\{0, e, 1\}$ an ultrafilter $U_{a}$ of $A$ containing $a$.

Now, for each $n \in \mathbb{N} \backslash\{0\}$ we define an embedding $f_{n}: \mathbf{G}_{n} \rightarrow \mathbf{G}_{n+1}$ such that (P1) and (P2) hold by

$$
\begin{aligned}
& f_{n}\left(x_{1}, \ldots, x_{n}\right)=\left(x_{1}, \ldots, x_{n}, x_{r(i)}\right) \quad \text { if } n=2 i \\
& f_{n}\left(x_{1}, \ldots, x_{n}\right)=\left\{\begin{array}{lll}
\left(x_{1}, \ldots, x_{n}, 1\right) & \text { if } x_{\pi_{2}(s(i))} \in U_{\pi_{1}(s(i))} \cup\{e\} & \text { if } n=2 i+1 \\
\left(x_{1}, \ldots, x_{n}, 0\right) & \text { otherwise } &
\end{array}\right.
\end{aligned}
$$

For the verification of (P1) consider an anti-atom $d=\left(d_{1}, \ldots, d_{n}\right)$ of $\mathrm{D}\left(\mathbf{G}_{n}\right)$. Let $j \in\{1, \ldots, n\}$ be such that $d_{j}=e$. By the infinity of $r^{-1}(j)$ there is $k>n / 2, k \in \mathbb{N}$, such that $r(k)=j$. Then, setting $\left(d_{1}, \ldots, d_{2 k}\right)=g_{n, 2 k}(d)$,

$$
\begin{aligned}
g_{n, 2 k+1}(d) & =f_{2 k} \circ g_{n, 2 k}(d) \\
& =f_{2 k}\left(d_{1}, \ldots, d_{2 k}\right) \\
& =\left(d_{1}, \ldots, d_{2 k}, d_{r(k)}\right) \\
& =\left(d_{1}, \ldots, d_{n+k}, d_{j}\right) \\
& =\left(d_{1}, \ldots, d_{n+k}, e\right)
\end{aligned}
$$

follows. Thus, $g_{n, 2 k+1}(d)$ is not an anti-atom of $\mathbf{G}_{n, 2 k+1}$ anymore.

For the verification of $(\mathrm{P} 2)$ consider $a=\left(a_{1}, \ldots, a_{n}\right) \in \operatorname{Sk}\left(\mathbf{G}_{n}\right) \backslash\{0,1\}$ and let $j \in\{1, \ldots, n\}$ be such that $0<a_{j}<1$. By the infinity of $s^{-1}\left(a_{j}, j\right)$ there is $k>n / 2, k \in \mathbb{N}$, such that $\pi_{1}(s(k))=a_{j}$ and $\pi_{2}(s(k))=j$. Then, setting $\left(a_{1}, \ldots, a_{2 k+1}\right)=g_{n, 2 k+1}(a)$, we have

$$
\begin{aligned}
g_{n, 2 k+2}(a) & =f_{2 k+1} \circ g_{n, 2 k+1}(a) \\
& =f_{2 k+1}\left(a_{1}, \ldots, a_{2 k+1}\right) \\
& =\left(a_{1}, \ldots, a_{2 k+1}, 1\right)
\end{aligned}
$$

The last equality holds because $a_{\pi_{2}(s(k))}=a_{j} \in U_{a_{j}}=U_{a_{\pi_{1}(s(k))}}$.

Claim The direct limit $\mathbf{G}$ of the directed family $\left\{\left\langle\mathbf{G}_{m}, g_{m, n}\right\rangle: m, n \in \mathbb{N}, 1 \leq\right.$ $m \leq n\}$ of p-semilattices is countable and existentially closed. 
Proof $\mathbf{G}$ is countable since a countable union of countable sets is countable. That $\mathbf{G}$ is algebraically closed follows from Theorem 1; Let $\mathbf{S}$ be a finite subalgebra of $\mathbf{G}$. By the construction of $\mathbf{G}$, there is an $n \in \mathbb{N}$ such that that the carrier $S$ of $\mathbf{S}$ is a subset of $G_{n}$. Therefore, there is a subalgebra $\mathbf{S}^{\prime}$ of $\mathbf{G}$ isomorphic to $\mathbf{G}_{n}=(\widehat{\mathbf{A}})^{n}$ extending $\mathbf{S}$.

By Theorem 2 it remains to show that $\mathbf{G}$ satisfies (EC1) $(\mathrm{EC} 5)$, (EC1) the order restricted to the skeletal elements is dense - is satisfied as it is satisfied in A. To prove axioms (EC2) (EC5) we denote for $x \in \bigcup_{n=1}^{\infty} G_{n}$ by $[x] \in G$ the equivalence class of $x$.

For (EC2) consider arbitrary $b_{1}, b_{2} \in \mathrm{Sk}(\mathbf{G})$ and $d \in \mathrm{D}(\mathbf{G})$ such that $b_{1} \leq b_{2}<d$ and $b_{1}^{*} \| d$. There is $n \in \mathbb{N}$ and $x=\left(x_{1}, \ldots, x_{n}\right), y=\left(y_{1}, \ldots, y_{n}\right)$, $w=\left(w_{1}, \ldots, w_{n}\right) \in G_{n}$ such that $b_{1}=[x], b_{2}=[y], d=[w], \operatorname{Sk}(x), \operatorname{Sk}(y)$, $\mathrm{D}(w), x \leq y<w$ and $x^{*} \| w$. We first assume that $w$ is not an anti-atom of $\mathbf{G}_{n}$. Then, without loss of generality, we can assume $x_{1}=0, w_{1}=w_{2}=e$. Then put $z=\left(1, z_{2}, 1, \ldots, 1\right)$ with $y_{2}<z_{2}<e$ to obtain $y<z, z \| w$, $x^{*} \wedge z \| w$ and $x \dot{\vee} z^{*}<w$. The last inequality follows from $x_{2} \leq y_{2}<z_{2}$, which implies $0<x_{2}^{*} \wedge z_{2}$, thus $e>\left(x_{2}^{*} \wedge z_{2}\right)^{*}=x_{2} \dot{\vee} z_{2}^{*}$. Putting $b_{3}=[z]$ yields the element requested in (EC2)

If $w$ is an anti-atom there is by (P1) an $l \in \mathbb{N}$ such that $g_{n, n+l}(w)$ is not an anti-atom of $\mathbf{G}_{n+l}$ anymore. For $x^{\prime}:=g_{n, n+l}(x), y^{\prime}:=g_{n, n+l}(y)$ and $w^{\prime}:=g_{n, n+l}(w)$ we find as above $z \in G_{n+l}$ such that $y^{\prime}<z, x^{\prime *} \wedge z \| w^{\prime}$ and $x^{\prime} \dot{\vee} z^{*}<w^{\prime}$. Putting $b_{3}=[z]$ then yields the element requested in (EC2) because $[x]=\left[x^{\prime}\right],[y]=\left[y^{\prime}\right],[w]=\left[w^{\prime}\right]$.

For (EC3) consider an arbitrary $b \in \operatorname{Sk}(\mathbf{G})$ with $b<1$. There is $n \in \mathbb{N}$ and $x=\left(x_{1}, \ldots, x_{n}\right) \in G_{n}$ such that $b=[x], \operatorname{Sk}(x)$ and $x<1$. By (P2) there is $k \in \mathbb{N}$ such that $\pi_{n+k}\left(g_{n, n+k}\left(x^{*}\right)\right)=1$. Then, with $(1, \ldots, 1, e) \in G_{n+k}$, we can choose $d=[(1, \ldots, 1, e)]$ as the element requested in (EC3).

For (EC4) consider arbitrary $d_{1}, d_{2} \in \mathrm{D}(\mathbf{G})$ such that $d_{1}<d_{2}$. There is $n \in \mathbb{N}$ and $x, y \in G_{n}$ such that $d_{1}=[x], d_{2}=[y]$. There are $l \in \mathbb{N}$ and $z \in \mathrm{D}\left(\mathbf{G}_{n+l}\right)$ such that $g_{n, n+l}(x)<z<g_{n, n+l}(y)$ : We have $x=\bigwedge_{j \in J_{x}} a_{j}$, $y=\bigwedge_{j \in J_{y}} a_{j}$ for subsets $J_{y} \subsetneq J_{x} \subseteq\{1, \ldots, n\}, a_{j}$ being an anti-atom of $\mathrm{D}\left(\mathbf{G}_{n}\right)$ for $j \in J_{x}$. For $j_{0} \in J_{x} \backslash J_{y}$ there is by (P1) a least $l \in \mathbb{N}$ such that $g_{n, n+l}\left(a_{j_{0}}\right)$ is not an anti-atom of $\mathbf{G}_{n+l}$ anymore, that is, there is are antiatoms $u_{1}, u_{2} \in G_{n+l}$ with $g_{n, n+l}\left(a_{j_{0}}\right)=u_{1} \wedge u_{2}$ and $g_{n, n+l}\left(a_{j}\right) \| u_{i}$ for all $j \in J_{y}, i=1,2$. Because $\mathrm{D}\left(\mathbf{G}_{n+l}\right)$ is boolean, that is, a $\wedge$-reduct of a boolean algebra, we obtain

$$
\begin{aligned}
g_{n, n+l}(x)=\bigwedge_{j \in J_{x} \backslash\left\{j_{0}\right\}} g_{n, n+l}\left(a_{j}\right) \wedge & u_{1} \wedge u_{2}<\bigwedge_{j \in J_{x} \backslash\left\{j_{0}\right\}} g_{n, n+l}\left(a_{j}\right) \wedge u_{1} \\
& \leq \bigwedge_{j \in J_{y}} g_{n, n+l}\left(a_{j}\right) \wedge u_{1}<\bigwedge_{j \in J_{y}} g_{n, n+l}\left(a_{j}\right),
\end{aligned}
$$

which implies

$$
g_{n, n+l}(x)<g_{n, n+l}(y) \wedge u_{1}<g_{n, n+l}(y) .
$$


We have

$$
d_{1}=[x]=\left[g_{n, n+l}(x)\right]<\left[g_{n, n+l}(y) \wedge u_{1}\right]<\left[g_{n, n+l}(y)\right]=[y]=d_{2},
$$

and we can choose $d_{3}=\left[g_{n, n+l}(y) \wedge u_{1}\right]$ as the element requested in (EC4).

For (EC5) consider arbitrary $b \in \mathrm{Sk}(\mathbf{G})$ and $d_{1} \in \mathrm{D}(\mathbf{G})$ such that $0<b<$ $d_{1}$. There is $n \in \mathbb{N}$ and $x=\left(x_{1}, \ldots, x_{n}\right), y=\left(y_{1}, \ldots, y_{n}\right) \in G_{n}$ such that $b=[x], d_{1}=[y], \operatorname{Sk}(x), \mathrm{D}(y), 0<x<y$. Let us assume that there is no $z \in \mathrm{D}\left(\mathbf{G}_{n}\right)$ such that $z<y, x \| z$ and $x^{*} \wedge y=x^{*} \wedge z$, since otherwise we put $d_{2}=[z]$.

By (P2) there is an $l \in \mathbb{N}$ such that $\pi_{n+l}\left(g_{n, n+l}(x)\right)=\pi_{n+l}\left(g_{n, n+l}(y)\right)=1$. Defining $z \in G_{n+l}$ by putting $z_{j}=\pi_{j}\left(g_{n, n+l}(y)\right)$ for $1 \leq j \leq n+l-1$ and $z_{n+l}=e$ we then can choose $d_{2}=[z]$ as the element requested in (EC5)

\section{References}

1. Adler, J.: The model companion of the class of pseudocomplemented semilattices is finitely axiomatizable. Algebra Universalis (2014), (DOI) 10.1007/s00012-014-0297-9

2. Balbes, R., Horn, A.: Stone lattices. Duke Mathematical Journal 38, 537-545, (1970)

3. Burris, S.: Model companions for finitely generated universal Horn classes, J. Symbolic Logic 49, 68-74, Vol. 1 (1984)

4. Burris, S., Werner, H.: Sheaf constructions and their elementary properties, Trans. Amer. Math. Soc., 48, 269-309 (1979)

5. Frink, O.: Pseudo-complements in semilattices. Duke Mathematical Journal 37, 505514, (1962)

6. Jones, G.: Pseudocomplemented semilattices. PhD thesis, UCLA (1972)

7. Macintyre, A.: Model completeness. In Barwise, J. (ed.) Handbook of Mathematical Logic, pp. 139-180 North-Holland, Amsterdam (1977)

8. Schmid, J.: Algebraically closed p-semilattices. Arch. Math., Vol. 45, 501-510 (1985)

9. Wheeler, W.: Model-companions and definability in existentially complete structures. Israel Journal of Mathematics 25, 305-330 (1976) 